This is a postprint version of the following published document:

Mikes, F., González-Benito, J., Serrano, B.,

Bravo, J., Baselga, J. (2002). Fluorescence monitoring of polymerization reaction. A new method for treating fluorescence experimental data. Polymer, 43 (16), pp. 4331-4339.

DOI: $10.1016 / \mathrm{S} 0032-3861(02) 00296-3$

(C) Elsevier, 2002

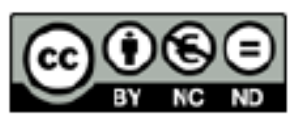

This work is licensed under a Creative Commons Attribution-NonCommercialNoDerivatives 4.0 International License. 


\title{
Fluorescence monitoring of polymerization reaction. A new method for treating fluorescence experimental data
}

\author{
F. Mikeš, F. González-Benito, B. Serrano, J. Bravo, J. Baselga* \\ Instituto Tecnológico Alvaro Alonso Barba, Universidad Carlos III de Madrid, Avda. de la Universidad 30, 28911 Leganés, Madrid, Spain \\ * Corresponding author. Tel.: +34-1-624-94-67; fax: +34-1-624-94-30. mail address: jbaselga@ing.uc3m.es (J. Baselga).
}

\begin{abstract}
A new method has been found for monitoring polymerization reactions in situ and in real time. The first moment of fluorescent emission, $\langle\nu\rangle=\sum I_{\mathrm{F}}(\nu) \nu / \sum_{\mathrm{F}} I_{\mathrm{F}}(\nu)$ is calculated from fluorescence spectra as a function of polymerization time and can be successfully correlated with the conversion of functional groups, obtained by an independent technique, with a very low level of experimental scatter. The statistical analysis of the method has been performed; some simple computer experiments allowed to study the influence of the most important experimental variables yielding the confidence interval of $\langle\nu\rangle$ as a function of the noise to signal ratio. This method was applied with stepwise polyaddition (epoxide curing) and polymerization by free radical mechanisms. 5-Dimethylaminonaphthalene-1-sulfonamide derivatives, 4dialkylamino-4'-nitrostilbene and pyrene were used as probes and/or labels. Other methods reported in the literature have been applied also. Comparison with them reveals that the first moment method is more reliable for monitoring polyaddition reactions.
\end{abstract}

\section{Keywords}

Fluorescent probes; Fluorescent labels; Epoxy resins

\section{Introduction}

In many cases, the degree of polymerization conversion must be strictly on-line controlled so as to achieve optimum material properties. Recently fluorescence techniques have been used to monitor the curing process of epoxide resins [1], polyurethanes [2] and different vinyl and acrylate/ methacrylate formulations. This field is therefore very active because of commercial applications and also because the interaction of fluorophore molecules in excited states with viscous liquids or solids opens new perspectives for the development of photorheology.

Fluorescence parameters that usually change with progressing epoxide curing reactions or the polymerization of double bonds are: fluorescence intensity, shift in emission maximum and half bandwidth. Three approaches have been applied to follow the cure of resins in situ: (a) the ratio of the fluorescence intensity of a probe to the intensity of a standard that does not change its fluorescence quantum yield with the progress of reaction [9], (b) the intensity ratio method [3-8] and (c) the shift in the emission maximum $[10,11]$. Using these three parameters some correlation can be obtained with the conversion degree regardless of the intensity of the excitation source, which is commonly the main drawback of fluorescence methods. Nevertheless, experimental noise may preclude its use in either research or industrial work.

The main objectives of this work can be formulated as follows: (a) To compare different methods for the evaluation of fluorescence data for the determination degree of conversion in different polymerization processes (leading to linear or crosslinked products); (b) To show that the first moment of the emission band $\langle\nu\rangle=\sum I_{\mathrm{F}}(\nu) \nu / \sum I_{\mathrm{F}}(\nu)$ can be successfully correlated with the conversion degree of functional groups with a very low level of noise; (c) To show that this method of analyzing fluorescence spectra can be used for very different fluorophores; (d) To perform statistical analysis of the method to validate it and to set the experimental conditions under which it can be used.

In this work we present results for the following systems: (a) Free radical polymerization of cyclohexyl methacrylate (CHMA) in presence of pyrene (Py, probe) and/or 1-pyrenemethyl methacrylate (MMA-Py, probe and label). (b) Curing of the stoichiometric reaction mixtures of diglycidyl ether bisphenol A (DGEBA) with $n$-butyl amine (BA, linear polymer), $N$-methylethylenediamine (MEDA, crosslinked polymer) and ethylenediamine 
(EDA, crosslinked polymers), monitored by 5-dimethylaminonaphthalene-1-sulfonamido (DNS) fluorophore that was used either as a probe or a label. (c) Curing reaction of DGEBA, labeled with trans-4-dialkylamino-4'-nitrostilbene (DANS) moiety, using EDA as well. Fluorescence data are analyzed by the intensity ratio method and by calculation of the first moment of the emission band $\langle\nu\rangle$. Furthermore the next correlation of these quantities and the emission maximum of fluorophores with the degree of conversion of either epoxy groups, $\alpha_{\mathrm{E}}$, or double bonds, $\alpha_{\mathrm{M}}$, determined by absolute methods, are established.

\section{Experimental}

\subsection{Low molecular weight compounds-probe and label precursors}

(a) N-(2-Aminoethyl)-5-dimethylamino-1-naphthalenesulfonamide (DNS-EDA) was prepared by the reaction of 5-dimethylamino-1-naphthalenesulfonyl chloride (DNSCl) with EDA [12]. DNS-Cl (Fluka) was used as received. In comparison with Ref. [12] $100 \mathrm{M}$ excesses of EDA over $\mathrm{DNS}-\mathrm{Cl}$ was used to minimize the amount of the disubstituted derivative. The product was homogeneous according to thin layer chromatography (TLC); $\mathrm{mp}$ 155.2 ${ }^{\circ} \mathrm{C} . \mathrm{C}_{14} \mathrm{H}_{19} \mathrm{~N}_{3} \mathrm{SO}_{2}\left(293.39 \mathrm{~g} \mathrm{~mol}^{-1}\right.$ ) Calcd: C, 57.31; H, 6.53; N, 14.32; S, 10.93. Found: C, 57.32; H, 6.56; N, 13.99; S, 11.03. UV (methanol) $\lambda_{\max }(\mathrm{nm})\left[\epsilon\left(1 \mathrm{~mol}^{-1}-\right.\right.$ $\left.\left.\mathrm{cm}^{-1}\right)\right]=282$ (1560), 338 (4400).

(b) $N$-(Di-n-butyl)-5-dimethylamino-1-naphthalenesulfonamide $(\mathrm{DNSd}-\mathrm{Bu})$ was prepared by the reaction of DNS-Cl $\left(1 \mathrm{~g}, 3.7 \times 10^{-3} \mathrm{~mol}\right.$, chloroform $\left.30 \mathrm{ml}\right)$ with excess of di- $n$-butylamine $\left(1.92 \mathrm{~g}, 1.48 \times 10^{-2} \mathrm{~mol}\right.$, chloroform $80 \mathrm{ml}$ ). The reaction mixture was extracted with water, several times with diluted hydrochloric acid ( $5 \mathrm{wt} \%)$, then with sodium hydrogen carbonate solution (5 wt \%) and finally with water. The chloroform solution was dried with anhydrous sodium sulfate. The residue after evaporation of the chloroform was recrystallized from the ethanol/water $(1 / 1, \mathrm{v} / \mathrm{v})$ mixture. The product was homogeneous according to TLC; $\mathrm{mp} 67^{\circ} \mathrm{C} . \mathrm{C}_{20} \mathrm{H}_{30} \mathrm{~N}_{2} \mathrm{O}_{2} \mathrm{~S}\left(362.54 \mathrm{~g} \mathrm{~mol}^{-1}\right)$ Calcd: C, 66.26; H, 8.34; N, 7.73; S, 8.83. Found: C, 66.40; H, 8.05; $\mathrm{N}, 7.70 ; \mathrm{S}, 9.11$.

(c) Trans-4-Amino-4'-nitrostilbene(trans-4-aminophenyl-4'-nitrophenylvinylen) (AmNST). Trans-4,4'-dinitrostilbene(trans-bis-(4-nitrophenyl)vinylen) was prepared by the reaction of 4-nitrobenzyl chloride with alcoholic sodium hydroxide $[13,14]$. The product was recrystallized five times from nitrobenzene and sublimated under high vacuum. mp $298.5{ }^{\circ} \mathrm{C}$ (Ref. [14]: $296-305^{\circ} \mathrm{C}$; Ref. [15]: 280-285 ${ }^{\circ} \mathrm{C}$ ). AmNST was prepared by reducing trans-4,4'-dinitrostilbene with polysulfide in ethanol [14]. The product was crystallized from nitrobenzene and further sublimation of the product under high vacuum was carried out. mp $249{ }^{\circ} \mathrm{C}$ (Ref. [14]: $245-245.5^{\circ} \mathrm{C}$ ). UV (methanol): $\lambda_{\max }=$
$403.3 \mathrm{~nm} \mathrm{C}_{14} \mathrm{H}_{12} \mathrm{~N}_{2} \mathrm{O}_{2}\left(240.26 \mathrm{~g} \mathrm{~mol}^{-1}\right)$ Calcd: C, 69.99; H, 5.03; N, 11.66. Found: C, 69.79; H, 5.06; N, 11.61.

(d) Trans-4-Dimethylamino-4'-nitrostilbene (DMANS) was prepared by adapting the procedure [16] used for the preparation of 4-dibutylamino- $4^{\prime}$-nitrostilbene. The crude product was purified by crystallization from toluene and by TLC.

(e) Other products. Py and MMA-Py (Molecular Probes, Inc.) were used as received. DGEBA (Aldrich) (molecular weight $348 \mathrm{~g} \mathrm{~mol}^{-1}$ ) was purified by recrystallization from acetone and methanol, carefully dried and stored under nitrogen; mp $42.5-43.6^{\circ} \mathrm{C}$. CHMA (Aldrich) was rectified prior to use at reduced pressure. BA (Aldrich) and MEDA (Aldrich) were refluxed over potassium hydroxide for $5 \mathrm{~h}$ and distilled. EDA (Aldrich) was used as received.

\subsection{Labeling of the DGEBA}

The DGEBA was labeled by reaction with DNS-EDA. In a typical labeling experiment the DGEBA (47.9620 g, $\sim 0.276$ mol epoxy groups) was heated to $60{ }^{\circ} \mathrm{C}$ while stirring with DNS-EDA $\left(0.0868 \mathrm{~g}, 2.959 \times 10^{-4} \mathrm{~mol}\right)$ for $6 \mathrm{~h}$. The efficiency of the labeling reaction was followed by TLC and size exclusion chromatography (SEC). Analogously, the DGEBA labeled with DANS moiety in the side chain was prepared by reaction of excess of DGEBA with AmNST at $160{ }^{\circ} \mathrm{C}$ for $16 \mathrm{~h}$. At the end of the reaction time, according to TLC and SEC, the reaction mixture contained neither starting DNS-EDA nor AmNST.

\subsection{Polymerization and curing reaction}

Polymerization of CHMA in presence of Py or MMAPy $\left(1 \times 10^{-4} \mathrm{~mol} \mathrm{l}^{-1}\right)$ was carried out under nitrogen at $60{ }^{\circ} \mathrm{C}$ using azobisisobutyronitrile $(1 \mathrm{wt} \%)$ as the initiator in a Perkin-Elmer DSC 7 apparatus. Details of the experimental arrangement and evaluation of $\alpha_{\mathrm{M}}$ by DSC were described elsewhere [17].

The details of curing of the stoichiometric reaction mixtures of DGEBA with BA, MEDA and/or EDA in the presence of the DNS label or probe and determination of $\alpha_{\mathrm{E}}$ by FTNIR were previously described [18].

\subsection{Fluorescence spectroscopy}

Emission spectra were taken on a Perkin-Elmer LS-50B spectrofluorimeter at certain cure time intervals and were corrected for the photomultiplier response. For monitoring the cure of epoxy systems the standard front face accessory was used. For monitoring the polymerization reaction of acrylic monomers, fluorimeter and DSC equipments were coupled using a bifurcated light guide; details are given in Ref. [17]. The wavelengths that were chosen for the application of the modified intensity ratio method represent the lowest and the highest intensity change that occurred during the polyaddition reaction (the emission spectrum of 
the reaction mixture taken at $\alpha_{\mathrm{E}}, \alpha_{\mathrm{M}} \rightarrow 1$ was divided by the spectrum of the reaction mixture at $\alpha_{\mathrm{E}}, \alpha_{\mathrm{M}} \rightarrow 0$ ). In the case of DNS fluorophore (label, probe), samples were excited at $350 \mathrm{~nm}$. For the DANS structural unit (label) excitation wavelength was the wavelength at maximum absorption, $460 \mathrm{~nm}$. In all cases the excitation and emission slits were equal. The values of the emission maxima were obtained using the Origin 4.1 standard routine for emission band integration.

\section{Results and discussion}

\subsection{Labeling of DGBA: UV/VIS and fluorescence spectra}

Unique reactivity of the primary and secondary amino groups with the epoxy groups justified the effort to prepare fluorescent labels containing primary amino groups. The epoxy groups react readily with the primary and secondary aliphatic amino groups and at a higher temperature with the primary and secondary aromatic amino groups as well. At high molar excess of epoxy groups over a fluorophore containing primary amino groups, the labeled molecule consists of two epoxy groups with fluorophore in the side chain (Scheme 1). In the case of AmNST, formation of the DANS structural unit was proved not only by TLC analysis of model reaction of phenyl glycidyl ether with AmNST, but also from UV/VIS absorption spectra. The absorption maxima of AmNST, DMANS, and labeled DGEBA in methanol are at 403.3, 425.7, and $424.2 \mathrm{~nm}$, respectively. Alkylation of the primary amino group in AmNST causes a red shift in the absorption maximum. On the other hand, substitution by an electron-withdrawing group brings about a large opposite spectral shift. For example, absorption maximum of trans- $\mathrm{N}$-4-(4-nitrostyryl)phenylmethacrylamide [19] in the same solvent is at $370 \mathrm{~nm}$.

The addition reaction of the primary amino group of DNS-EDA to the epoxy group does not shift the absorption of the dansyl fluorophore-electron density of the naphthalene ring remains unchanged.

The DNS label and probe used in the aforementioned epoxide resin formulations cover excitation spectral range $320-380 \mathrm{~nm}$. Due to the possible overlapping of the UV/VIS absorption bands of the epoxide reaction mixtures and the fluorescence label or probe, their absorption UV/VIS spectra were measured. Two important conclusions can be drawn: (1) Negligible changes in the absorption spectra were observed in the long wavelength region (300$380 \mathrm{~nm}$ ) for labeled and unlabeled reaction mixtures throughout the curing progress (the extent of the epoxy group conversion $0-0.80)$; (2) The stoichiometric DGEBA-EDA and/or MEDA reaction mixture cut off absorption is at around $335 \mathrm{~nm}$ and shifts to $350 \mathrm{~nm}$ with progress of the curing $\left(\alpha_{\mathrm{E}}=0.65\right)$. The same reaction mixtures labeled with the DNS fluorophore strongly absorb between 310 and $390 \mathrm{~nm}$. This absorption is caused by the

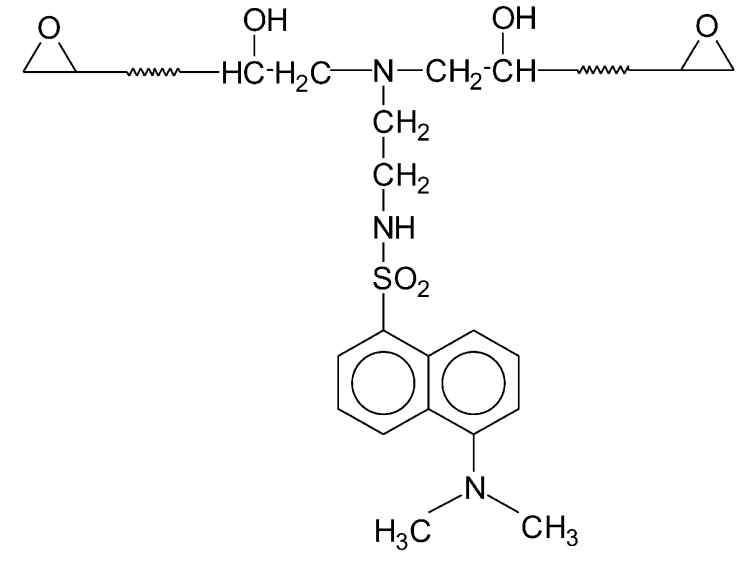

Scheme 1

DNS fluorophore. To eliminate emission of the DGEBAEDA and/or MEDA reaction mixtures, a relatively high concentration of the DNS fluorophore was used. Therefore, the fraction of light absorbed by the DNS fluorophore is close to unity and observed emission in curing experiments only represents the emission of DNS fluorophore. The reabsorption of the emitted radiation in spite of rather high optical density, did not take place owing to a large Stoke's shift of the DNS fluorophore in these reaction media.

The UV/VIS absorption of DANS fluorophore in the DGEBA-EDA reaction mixture is shifted farther in the visible region. The absorption maximum is at $\sim 460 \mathrm{~nm}$. In this case the excitation radiation is absorbed by DANS structural units only. Possible partial reabsorption of the emitted radiation due to a smaller Stoke's shift in this case may distort the blue edge of the emission spectrum. However, it has been shown that a five-times decrease in the concentration of the DANS fluorophore moiety in this reaction mixture does not affect the course of the dependence of $\langle\nu\rangle$ on the epoxy groups conversion $\alpha_{\mathrm{E}}$ shown in Fig. 3.

\subsection{The monitoring of polymerization processes}

Generally, the run-to-run reproducibility of the profile shape of the fluorescence intensity signal is good. However, the reproducibility of the absolute intensity values is unsatisfactory. This problem is typical for the front-face experimental setup of taking emission spectra especially for solid samples. The run-to-run variations in the fluorescence intensities are caused by the differences in the mutual positions of the sample (even with 'perfect' tool-mounted light guides which permit fluorescence excitation and emission to be coupled) and excitation beam. The differences in the surface roughness of the small area 'viewed' by the emission entrance slit as well as the stability of the excitation source also plays an important role. For solvatochromic probes, changes in the fluorescence maximum wavelength as a function of the conversion degree produce a highly characteristic signal profile that is 
reproducible and sometimes also reveals the main chemical transformations. The molecular origin of the changes in the emission maximum is complex in this case because both the dielectric environment of the fluorophores and the mobility of the dipoles surrounding the fluorophores and fluorophore itself change during polyaddition reactions.

In the intensity ratio method originally proposed by Neckers [3-7], this ratio is plotted vs. conversion of functional groups as determined by an absolute method. In a modified method [2] the normalized ratio, $R / R_{0}$, of the fluorescence intensities at two wavelengths is calculated as a function of reaction time. The magnitude $F$ defined as $F=1$ $-R / R_{0}$, where $R$ and $R_{0}$ are the intensity ratios at reaction time $t$ and at $t=0$, respectively, is correlated with conversion.

The weighted average wavenumber $\langle\nu\rangle$ is a well-known parameter used in photophysics [20]. It corresponds to the first moment of emission spectrum of a given fluorophore and has been used for studying the fluorescence shift of the DNS fluorophore in binary solvents [21]. The dependence of $\langle\nu\rangle$ together with $F$ and EM on $\alpha_{\mathrm{E}}$ is shown in Fig. 1(a) and (b) for DNS probe and DNS label, respectively, in the DGEBA-BA mixture at $40{ }^{\circ} \mathrm{C}$. In Fig. 2(a) and (b) the same dependencies are depicted for DNS labeled DGEBAMEDA and/or EDA reaction mixtures, respectively. Results for the same system DGEBA-EDA at $40{ }^{\circ} \mathrm{C}$, but labeled with DANS, are depicted in Fig. 3, and in Fig. 4(a) and (b) for polymerization of CHMA by free radical mechanism in presence of Py and MMA-Py, respectively.

In all cases the dependence of the emission maximum on $\alpha_{\mathrm{E}}$ is characterized by rather large scatter in emission maximum values. It is easy to see that there is a fundamental difference between the dependence of the emission maximum and $\langle\nu\rangle$ when plotted vs. $\alpha_{\mathrm{E}}$. In most cases the dependence of $\langle\nu\rangle$ is smooth, allowing one to use this dependence for the determination of $\alpha_{\mathrm{E}}$ on-line and in real time with a relatively high accuracy. When comparing Fig. 1(a) and (b), one sees that the DNS probe is very sensitive to conversion changes in the range of 0.05 up to 0.25 and the DNS label in conversion interval 0.2-1.0. Remarkable differences between fluorescence responses have been observed for the DNS and DANS labels in the curing system DGEBA-EDA. The DNS label can be used for estimation of $\alpha_{\mathrm{E}}$ up to $\alpha_{\mathrm{E}} \sim 0.55$, but one cannot distinguish between $\alpha_{\mathrm{E}} \sim 0.55-0.77$ owing to a nearly constant value of $\langle\nu\rangle$. On the other hand, DANS can monitor the curing reaction with very good precision up to the final conversion reached in the experiment. In addition, changes in the slope of the dependence indicate [18] the onset of tertiary amino groups formation $\left(\alpha_{\mathrm{E}} \sim 0.2\right)$, the gel point $\left(\alpha^{\text {gel }}=\mathrm{E}\right.$ $0.58)$, and entry of the system to the glassy state $\left(\alpha_{\mathrm{E}} \sim 0.72\right)$.

There are at least two important differences in these two methods. First, a lower sensitivity of the intensity ratio method at higher degrees of conversion contrasts with the same dependence of $\langle\nu\rangle$. Second, modified intensity ratio method ( $\left.1-R / R_{0}\right)$ requires a normalization procedure
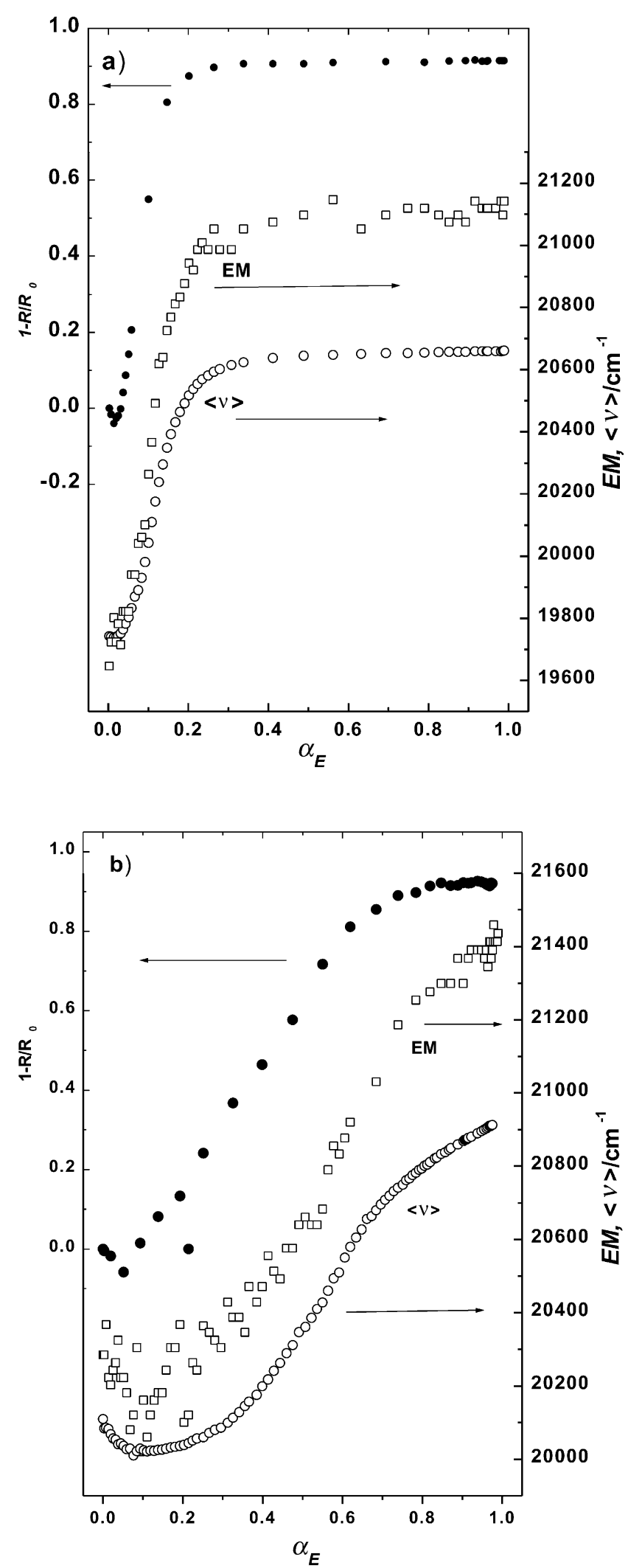

Fig. 1. Dependence of the reduced fluorescence intensity ratio $\left(1-R / R_{0}\right)$ emission maximum (EM) and the first moment of fluorescence emission band $(\langle\nu\rangle)$ on the epoxy groups conversion $\left(\alpha_{\mathrm{E}}\right)$ for the DGEBA-BA system at $40{ }^{\circ} \mathrm{C}$. (a) DNS probe, and (b) DNS label. Concentration of the DNS label and DNS probe $5.09 \times 10^{-3} \mathrm{~mol} \mathrm{~kg}^{-1}$ of the reaction mixture. 

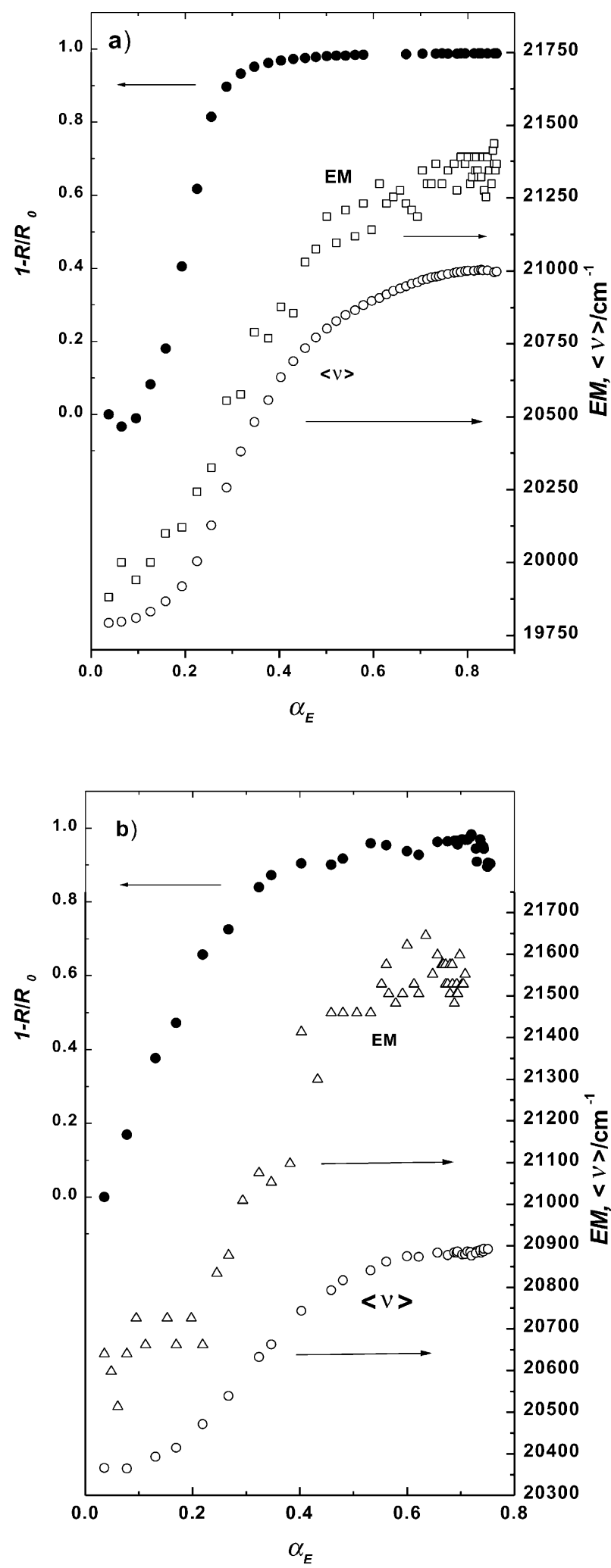

Fig. 2. Dependence of the reduced fluorescence intensity ratio $\left(1-R / R_{0}\right)$, emission maximum (EM) and the first moment of fluorescence emission band $(\langle\nu\rangle)$ on the epoxy groups conversion $\left(\alpha_{\mathrm{E}}\right)$ for DNS label in the (a) DGEBA-MEDA, and (b) DGEBA-EDA systems at $40^{\circ} \mathrm{C}$. Concentration of the DNS label was $5.39 \times 10^{-3}$ and $5.67 \times 10^{-3} \mathrm{~mol} \mathrm{~kg}^{-1}$ of the reaction mixture for (a) and (b), respectively.

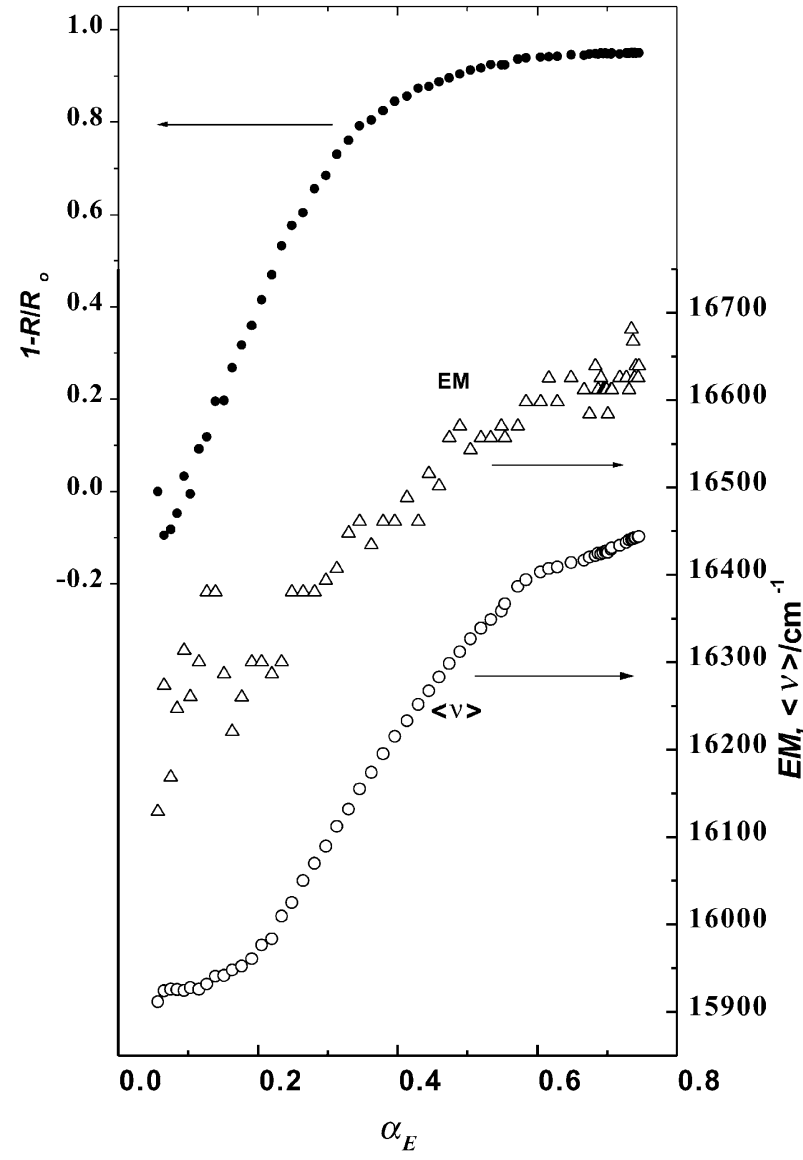

Fig. 3. Dependence of the reduced fluorescence intensity ratio $\left(1-R / R_{0}\right)$, emission maximum (EM) and the first moment of fluorescence emission band $(\langle\nu\rangle)$ of DANS label on the epoxy groups conversion $\left(\alpha_{\mathrm{E}}\right)$ for the DGEBA-EDA system at $40{ }^{\circ} \mathrm{C}$. Concentration of the DANS label $1.30 \times 10^{-3} \mathrm{~mol} \mathrm{~kg}^{-1}$ of the reaction mixture.

using the $R_{0}$ (at $t=0$ ) value, and this is experimentally difficult. Neither Neckers' original intensity ratio method nor the evaluation of $\langle\nu\rangle$ requires analysis of the emission spectrum at reaction time $t=0$.

Moreover, the intensity ratio method used for the determination of $\alpha_{\mathrm{E}}$ suffers from the same limitations as the method that uses the experimentally determined dependence of the fluorophore emission maximum on the epoxy groups conversion. The intensity ratio method requires: (a) A shift in the emission maximum of the fluorophore with increasing conversion of the functional groups; the larger the shift the more accurate and sensitive this method is. (b) For no shift in the emission maximum with the curing progress, either a change in the band shape or a change in other emission bands of the fluorophore are necessary. A case in which no shift is expected is the Py fluorophore. For Py the dependence of $\langle\nu\rangle$ on $\alpha_{\mathrm{M}}$ is in both cases smoother than similar dependence for $F$ (Fig. 4(a) and (b)). Polymerization of CHMA can be monitored with very good accuracy to the highest conversions using MMA-Py in the reaction mixture.

Clearly, even though the intensity method is simple and 

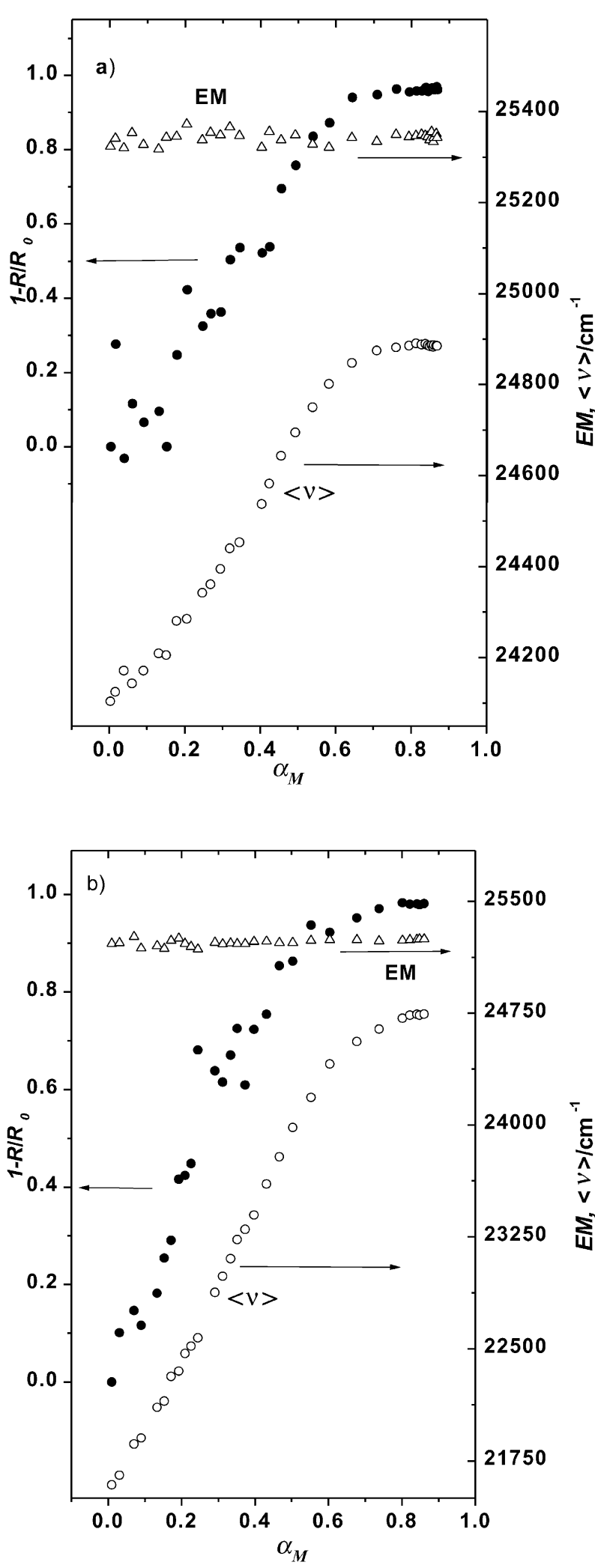

Fig. 4. Dependence of the reduced fluorescence intensity ratio $\left(1-R / R_{0}\right)$, emission maximum (EM) and the first moment of fluorescence emission band $(\langle\nu\rangle)$ on the double bonds conversion $\left(\alpha_{\mathrm{M}}\right)$ for polymerization of CHMA in the presence of (a) Py, and (b) MMA-Py at $60^{\circ} \mathrm{C}$. Concentration of Py fluorophore $1 \times 10^{-4} \mathrm{~mol} \mathrm{~kg}^{-1}$. fast at predicting $\alpha$, the estimation of $\alpha_{\mathrm{E}}$ or $\alpha_{\mathrm{M}}$ from $\langle\nu\rangle$ vs. $\alpha$ plot seems to be better. The computed $\langle\nu\rangle$ is insensitive to minor random fluctuations in the emission intensity profile as obtained from fluorimeters. The $\left\langle\nu^{2}\right\rangle^{1 / 2}$, other moments and the standard deviation of the emission spectra have been evaluated and plotted vs. $\alpha_{\mathrm{E}}$, but these plots do not differ significantly from that for $\langle\nu\rangle$.

\subsection{Statistical analysis of the $\langle\nu\rangle$ method}

An adequate use of the method requires the previous knowledge of the limits of application and an estimation of the uncertainty of the obtained $\langle\nu\rangle$ values. Obviously it is not possible to measure a statistically significant number of spectra to analyze the most important experimental variables such as emission band position, bandwidth, intensity and amount of noise. Instead, we have approached the problem making computer experiments. For doing so it is necessary to generate different synthetic spectra and to add different levels of noise.

A Gaussian function, $g\left(\lambda, \lambda_{\mathrm{C}}, \omega_{\lambda}\right)$, was used in the wavelength range $\lambda=200-900 \mathrm{~nm}$, as a reasonable equation for spectra generation [22]. Spectra which generated peaking at $\lambda_{\mathrm{C}}=450$ and $600 \mathrm{~nm}$, were common fluorophores present emission maxima. For each $\lambda_{\mathrm{C}}$, three bandwidths were explored $\omega_{\lambda}=40,80$, and $120 \mathrm{~nm}$. Each spectrum contained 1400 points and was converted into the wavenumber scale, $f\left(\nu, \nu_{\mathrm{C}}, \omega_{\nu}\right)$, using the common change equations:

$$
\nu\left(\mathrm{cm}^{-1}\right)=10^{7} \lambda^{-1}(\mathrm{~nm}) \quad f\left(\nu, \nu_{\mathrm{C}}, \omega_{\nu}\right)=\lambda^{2} g\left(\lambda, \lambda_{\mathrm{C}}, \omega_{\lambda}\right)
$$

Two sources of noise were considered [22]: (a) a dark noise, $B$, due to uncorrelated signals, and (b) Poisson noise, $P$, associated to the photon counting system. $B$ depends on the temperature sensitivity of the PMT and on the spurious leakage of room light. According to our experience it is common to find a random value between 0 and $100 \mathrm{c} \mathrm{s}^{-1}$ for $B$.

Before adding Poisson noise, reference spectra, $F^{0}\left(\nu, \nu_{\mathrm{C}}, \omega_{\nu}\right)$, were obtained normalizing $f\left(\nu, \nu_{\mathrm{C}}, \omega_{\nu}\right)$, multiplying it by an intensity factor, $I$, that ranged between $5 \times 10^{2}$ and $2 \times 10^{5}$ and rounding it to the nearest integer to simulate the photon counting detector response. Eq. (1) is the algorithm used.

$F^{0}\left(\nu, \nu_{\mathrm{C}}, \omega_{\nu}\right)=\operatorname{int}\left[\langle B\rangle+\frac{f\left(\nu, \nu_{\mathrm{C}}, \omega_{\nu}\right)}{\max \left[f\left(\nu, \nu_{\mathrm{C}}, \omega_{\nu}\right)\right]} \times I\right]$

where $\langle B\rangle$ is the average dark noise (for this case it was considered equal to $50 \mathrm{c} \mathrm{s}^{-1}$ ) and int $[i]$ and $\max [k]$ are functions that provide the nearest integer and the maximum value of the arguments $i, k$, respectively. The rounding off function, int $[i]$, was necessary to simulate the response of a photon counting detector.

To account for Poisson noise, it was considered a random value within the range $\pm n\left\{F^{0}\left(\nu, \nu_{\mathrm{C}}, \omega_{\nu}\right)\right\}^{1 / 2}$, which is equivalent to consider $2 n$ times the standard deviation of a 
Poisson distribution. Three values of $n$ were explored $n=1$, 5 , and 10 . Therefore, the synthetic spectra $S\left(\nu, \nu_{\mathrm{C}}, \omega_{\nu}\right)$, were obtained according to algorithm (2)

$$
\begin{aligned}
S\left(\nu, \nu_{\mathrm{C}}, \omega_{\nu}\right)= & F^{0}\left(\nu, \nu_{\mathrm{C}}, \omega_{\nu}\right)-\langle B\rangle+B+P \\
= & F^{0}\left(\nu, \nu_{\mathrm{C}}, \omega_{\nu}\right)-\langle B\rangle+\operatorname{int} \\
& \times\left[\operatorname{rnd}(0 ; B)+\operatorname{rnd}\left( \pm n\left\{F^{0}\left(\nu, \nu_{\mathrm{C}}, \omega_{\nu}\right)\right\}^{1 / 2}\right)\right]
\end{aligned}
$$

where $\operatorname{rnd}(i ; j)$ is the randomization function, that provides a random value between the arguments $i, j$.

The $\langle\nu\rangle^{S}$ parameter of the synthetic spectra were calculated as

$\langle\nu\rangle_{\nu_{\mathrm{C}}, \omega_{\nu}}^{S}=\frac{\int_{\nu_{1}}^{\nu_{2}} \nu S\left(\nu, \nu_{\mathrm{C}}, \omega_{\nu}\right)}{\int_{\nu_{1}}^{\nu_{2}} S\left(\nu, \nu_{\mathrm{C}}, \omega_{\nu}\right)}$

Although the definition of the first moment of a distribution requires integration between $-\infty$ and $+\infty$, in practice this is not possible because of the interference of the excitation band and of the harmonics of the monochromators. Furthermore, for a given $S$, centered at $\nu_{\mathrm{C}}$, integration limits should be large enough to account for the information contained in the high and low energy tails of the spectrum but should also be small enough to avoid the influence of the background noise, specially at high energy values.

To calculate the optimum value of $\nu_{1}$ and $\nu_{2}$, the following computer experiment was performed. A new simulation parameter, $\alpha$, was defined to set the integration limits as follows

$$
\nu_{1}=\nu_{1,1 / 2}+\alpha \nu_{1,1 / 2} \quad \nu_{2}=\nu_{1,1 / 2}-\alpha \nu_{1,1 / 2}
$$

where $\nu_{1,1 / 2}$ and $\nu_{2,1 / 2}$, are the wavenumbers at the halfheight of the spectrum, being $\omega_{\nu}=\nu_{1,1 / 2}-\nu_{2,1 / 2} . \alpha$ is related with the number of bandwidths contained within the integration limits according to Eq. (5):

$$
\frac{\nu_{1}-\nu_{2}}{\omega_{\nu}}=1+\alpha \frac{\nu_{1,1 / 2}-\nu_{2,1 / 2}}{\omega_{\nu}}
$$

Although $\alpha$ was used for simulation purposes, $\left(\nu_{1}-\nu_{2}\right) / \omega_{\nu}$ can be visualized more easily and will be used for presenting and discussing results.

A set of three Gaussian functions peaking at $450 \mathrm{~nm}$ and with bandwidths $\omega_{\lambda}=40,80$ and $120 \mathrm{~nm}$ were generated. $F^{0}$ was calculated according to algorithm (1). $S$ was calculated according to algorithm (2) using simulation parameters $I=40000 \mathrm{c} \mathrm{s}^{-1}, n=1, B=100 \mathrm{c} \mathrm{s}^{-1}$. $\alpha$ was varied in the range $\alpha=0-0.6$. For each bandwidth and each $\alpha$ value, $10^{3}$ spectra were generated, to obtain a statistically representative population, and the value of $\langle\nu\rangle^{S}$ was calculated correspondingly.

For a given set of simulation conditions it was possible to calculate the difference $\left[\langle\nu\rangle^{F^{0}}-\langle\nu\rangle^{S}\right]$ and its standard deviation. In Fig. 5, the standard deviation of this difference is plotted as a function of the number of bandwidths contained within the integration limits. A minimum is observed in all the three curves in which $\sigma$ ranges between 0.5 and $1 \mathrm{~cm}^{-1}$, representing that for each bandwidth there is an optimum integration range in which the found $\langle\nu\rangle^{S}$ and the expected $\langle\nu\rangle^{F^{0}}$ are very similar. For those minimums, the integration limits range between 2.75 and 3.1 times the bandwidth. Since the slope of the curves at their right hand side is very small and since the obtained optimum values for the different bandwidths are relatively similar it was assumed in the subsequent analysis a constant value of $\left(\nu_{1}-\nu_{2}\right) / \omega_{\nu}=3$.

Once the integration limits were set, a second computer experiment was performed to evaluate the sensitivity of the $\langle\nu\rangle$ parameter to changes in the important experimental variables: peak position, intensity, bandwidth and amount of Poisson noise. Preliminary computer testing showed that results for different bandwidths were very similar so this variable was excluded from this analysis and experiments were performed with a constant value of $\omega_{\lambda}=80 \mathrm{~nm}$. For each combination of the simulation variables $\left(\nu_{\mathrm{C}}, I, N\right), 10^{3}$ spectra were generated and the differences $\left[\langle\nu\rangle^{F^{0}}-\langle\nu\rangle^{S}\right]$ were calculated. It was checked that the population of the differences followed a normal distribution. Therefore, 1.96 times the standard deviation of the distribution of the differences provides the $95 \%$ confidence interval.

It was found that plotting $\pm 1.96 \sigma\left[\langle\nu\rangle^{F^{0}}-\langle\nu\rangle^{S}\right]$ against the nominal noise to signal ratio $(B / I)$ in a logarithmic scale was a convenient way for presenting results and allows drawing important conclusions about the accuracy and limits of the proposed method. The plots corresponding to a band centered at $450 \mathrm{~nm}$ are presented in Fig. 6(a) and the plots corresponding to a band centered at $600 \mathrm{~nm}$ in Fig. 6(b).

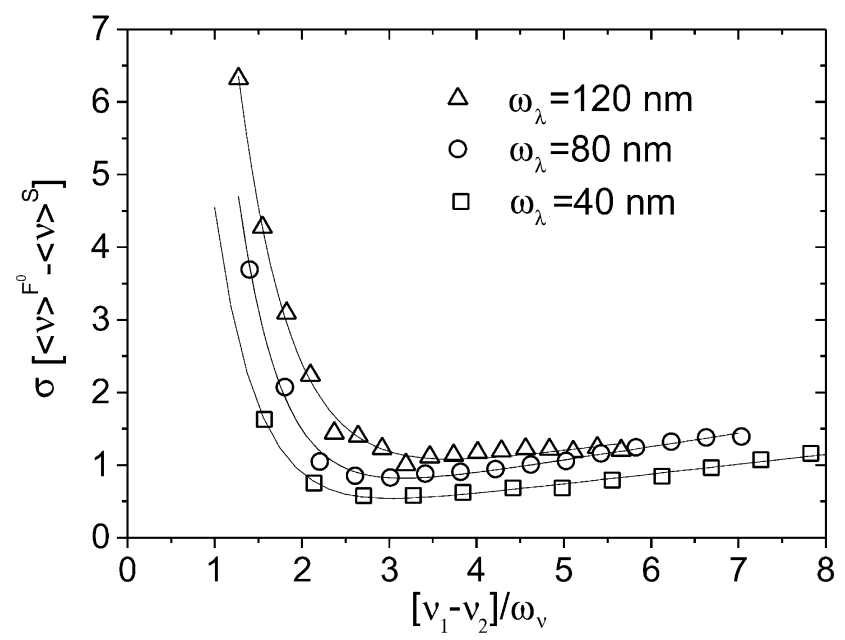

Fig. 5. Standard deviation of the difference $\left[\langle\nu\rangle^{F^{0}}-\langle\nu\rangle^{S}\right]$ as a function of the number of bandwidths contained within the integration limits $\left(\nu_{1}-\right.$ $\left.\nu_{2}\right) / \omega_{\nu}$ for three bands peaking at $450 \mathrm{~nm}$ and having three different bandwidths of 40,80 and $120 \mathrm{~nm}$. Population $=10^{3}$ spectra; $I=$ $410^{4} \mathrm{c} \mathrm{s}^{-1}, B=100 \mathrm{c} \mathrm{s}^{-1}, n=1$ (see text for explanation of symbols). 

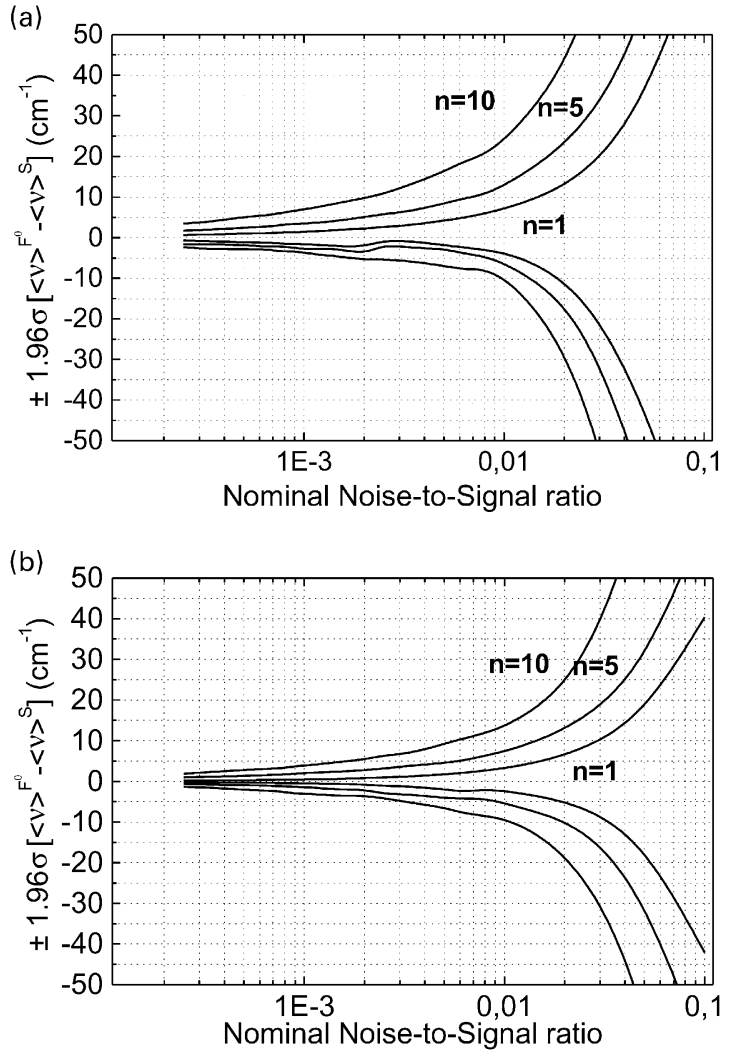

Fig. 6. Plot of the $95 \%$ confidence interval $(1.96 \sigma)$ for the standard deviation of the difference $\left[\langle\nu\rangle^{F^{0}}-\langle\nu\rangle^{S}\right]$ as a function of the noise to signal ratio at three different levels of Poisson noise $n=1,5$ and 10 (see text for details). Simulation conditions: $\omega_{\lambda}=80 \mathrm{~nm}$, population $=10^{3}$ spectra, (a) $\lambda_{\mathrm{C}}=450 \mathrm{~nm}$, and (b) $\lambda_{\mathrm{C}}=600 \mathrm{~nm}$.

It can be observed in both figures that the confidence intervals diverge almost exponentially as the noise to signal ratio increases and converge asymptotically to zero for very high signal levels. This behavior is more pronounced when the amount of Poisson noise is increased from $n=1$ to 10 . In comparing Fig. 6(a) and (b), it can also be observed that for emission bands appearing in the blue side of the spectrum the uncertainties are bigger than for red shifted bands.

Fig. 6(a) and (b) may provide the experimental conditions under which spectra should be obtained for achieving the minimum uncertainty in the determination of $\langle\nu\rangle$. It may be instructive to compare the resolution with which the wavelength at the maximum, $\lambda_{\mathrm{C}}$, can be obtained and its corresponding $\langle\nu\rangle$ value. For a low noise band centered in the range $450-600 \mathrm{~nm}$, the minimum absolute error in $\lambda_{\mathrm{C}}$ is usually about $\pm 0.5 \mathrm{~nm}$ (graphically determined) with common equipments and for common fluorophores. This value is about one order of magnitude higher than the standard error in the corresponding value of $\langle\nu\rangle$ (for $n=1$ and $B / I<0.01$ ).

In summary, the statistical analysis of the influence of common experimental variables on the value of $\langle\nu\rangle$, allows to draw the following conclusions: (a) Determination of $\langle\nu\rangle$ can be done with a much greater accuracy than $\lambda_{\mathrm{C}}$ or $\nu_{\mathrm{C}}$. (b) For an accurate calculation of $\langle\nu\rangle$ the amount of Poisson noise should be kept at a low level (several quick scans or a single scan at low monochromator speed) to reach a value of $n=1$. (c) The background noise should be kept also at low level (cooled PMT) to reach a value of $B<100 \mathrm{c} \mathrm{s}^{-1}$. (d) The fluorescence intensity should be maximized (for example, adjusting emission slit widths and concentration of high quantum yield chromophores) to reach a value of $I>10^{4} \mathrm{c} \mathrm{s}^{-1}$. (e) Excitation slit should be adjusted to minimize the influence of excitation light; otherwise, the integration limits should be rechecked. (f) Integration limits can be set at three times the bandwidth and should be recalculated for each spectrum.

With a carefully planned experiment taking into account the earlier mentioned points, the value of $\langle\nu\rangle$ can be easily calculated with a standard error of about $\pm 2 \mathrm{~cm}^{-1}$ which should be independent of either the fluctuations of the exciting light and on small variations on the position of the sample with respect to the excitation and emission slits.

\section{Conclusions}

According to our findings the first moment of the emission band evaluated from the emission spectra has been successfully correlated with the degree of functional groups conversion. This method was applied to epoxide curing and to polymerization of CHMA by free radical mechanism. This dependence can be used for the estimation of the conversion degree independently of the excitation source stability or the reaction mechanism and in most cases with higher accuracy than previously reported.

The method can be applied to different fluorescence probes and labels characterized by emission spectra possessing broad structureless bands (DNS), partially structured bands (DANS) or highly structured emission bands (Py) and regardless of the presence of the solvatochromic effect or not.

From the chemical point of view, the most important factor for an accurate determination of the degree of the functional groups conversion when monitoring the fluorescence response is the proper choice of the fluorophore (probe or label is sensitive enough in the entire range of conversions) for a particular system.

From the experimental point of view, the most important factors for an accurate determination of $\langle\nu\rangle$ are the noise to signal ratio and an adequate selection of integration limits.

\section{Acknowledgments}

Authors wish to thank European Commission (BE97-4472) and CAM (07N/0002/1998) for financial support. 


\section{References}

[1] Song JC, Sung CSP. Macromolecules 1993;26:4818.

[2] Vatanparast R, Li S, Hakala K, Lemmetyinen H. Macromolecules 2000;33:438

[3] Loufty RO. JPolym Sci, Polym Phys Ed 1982;20:825.

[4] Song JC, Torres-Filho A, Neckers DC. Radtech Proc 1994;1:338.

[5] Song JC, Neckers DC. Polym Engng Sci 1996;36(3):394.

[6] Jager WF, Volkers AA, Neckers DC. Macromolecules 1995;28:8153.

[7] Paczkowski J, Neckers DC. Macromolecules 1992;25:548.

[8] Peinado C, Salvador EF, Baselga J, Catalina F. Macromol Chem Phys 2000;.

[9] Wang FW, Lowry RE, Fanconi BM. Polymer 1986;27:1529.

[10] Jager WF, Kudesheva D, Neckers DC. Macromolecules 1996;29: 7351.

[11] Paik HJ, Sung NH. Polym Engng Sci 1994;34(12):1025.

[12] Nilson JL, Stenberg P, Ljunggren CH. Acta Pharm Suec 1971;8:497.

[13] Hanna SB, Iskander Y, Riad Y. J Chem Soc 1961;217.

[14] Calvin M, Buckles RE. J Am Chem Soc 1940;62:3324.

[15] Walden P, Kernfan A. Chem Ber 1890;23:1959.

[16] Ulman A, Willand CS, Kôhler W, Robello DR, Williams DJ, Handley L. J Am Chem Soc 1990;112:7083.

[17] Serrano B, Levenfeld B, Bravo J, Baselga J. J Polym Engng Sci 1996; $36: 175$.

[18] Mikeš F, Gonzalez-Benito FJ, Baselga J. J Macromol Sci Phys 2001;

[19] Mikeš F, Štrop P, Kálal J. Makromol Chem 1974;175:2375.

[20] Berlman IB. Handbook of fluorescence spectra of aromatic molecules. New York: Academic Press; 1971. p. 27.

[21] Leezenberg PB, Frank CW. Macromolecules 1995;28:7407.

[22] O'Connor DV, Phillips D. Time-correlated single photon counting. New York: Academic Press; 1984. p. 158 and 189. 